\title{
Cristalización de criolita a partir de un fundido: Rasgos macroscópicos y estructurales
}

\author{
Ma V. LÓPEZ-ACEVEDO, M. BENJOUALI, S. LÓPEZ ANDRÉS \\ Dpto. de Cristalografía y Mineralogía. Fac. de CC Geológicas. UCM. 28040 Madrid
}

\begin{abstract}
Al sobreenfriar un fundido de criolita $\left(\mathrm{Na}_{3} \mathrm{AlF}_{6}\right)$ se ha obtenido un agregado granular de ésta que se interpreta como un fenómeno de nucleación homogénea primaria. Los granos están cementados por una matriz en la que se concentran los elementos contaminantes del sistema (ej. $\mathrm{Si}$ ), debido a la presión de cristalización que ejerce la criolita durante su desarrollo. Las maclas polisintéticas que abundan en los diferentes granos se interpretan como una evidencia experimental de la transición ferroelástica que tiene lugar al disminuir la temperatura en el transcurso del proceso.
\end{abstract}

Palabras clave: criolita, crecimiento a partir de un fundido, transición ferroelástica, presión de cristalización, nucleación homogénea.

Cryolite crystallization from a melt: macroscopic and structural features

Overcooling of a cryolite $\left(\mathrm{Na}_{3} \mathrm{AlF}_{6}\right)$ melt gave rise to a granular aggregate. This aggregate can be regarded as the result of a process of homogeneous primary nucleation. Due to the crystallization pressure exerted by the cryolite during cooling, the grains are progressively embedded by a matrix constituted by the contaminant elements (e.g., Si) of the system. Abundant polysinthetic twins are here interpreted as an experimental evidence of processes related to the ferroelastic transition, that took place during cooling.

Key words: cryolite, melt growth, ferroelastic phase transition, crystallization pressure, homogeneous nucleation.

\section{INTRODUCCIÓN}

El nombre de este mineral, de fórmula $\mathrm{Na}_{3} \mathrm{AlF}_{6}$, hace alusión a su aspecto de hielo. Aunque se trata de un mineral poco común, sus propiedades como fundido han sido objeto de múltiples estudios desde el descubrimiento de su importante papel en el proceso Hall-Heroult (1885) para la obtención comercial del aluminio metal. En dicho proceso la criolita se utiliza como electrólito, para reducir las menas alumínicas (1). Su forma sólida, sin embargo, permanece relativamente desconocida y solo recientemente, ha comenzado a ser estudiada en profundidad. Posee una estructura de tipo perowskita, de gran actualidad tanto desde el punto de vista geológico como para la ciencia de los materiales. Además exhibe una conducta muy especial, con transiciones de fase y movilizaciones atómicas asociadas a ellas, que son responsables de sus peculiares propiedades mecánicas y eléctricas (2). Por encima de los $450^{\circ} \mathrm{C}$ se comporta como un flujo plástico que podría ser la explicación de las brechas del yacimiento de Ivigtut (Groenlandia), donde criolita pura constituye el cemento de los clastos de la roca (Ivigtut ha sido el depósito industrial mas grande del mundo, en la actualidad prácticamente agotado) (3). Entre esta temperatura y $\operatorname{los} 550^{\circ} \mathrm{C}$, el valor de su resistividad eléctrica responde al de los conductores superiónicos (4).

La estructura de la criolita $\left(2\left[\mathrm{Na}^{+}\left(\mathrm{Na}^{+}{ }_{0.5} \mathrm{Al}^{3+}{ }_{0.5}\right) \mathrm{F}_{3}\right]\right)$ se puede definir como una alternancia de octaedros $\left[\mathrm{AlF}_{6}\right]$ y $\left[\mathrm{Na}(1) \mathrm{F}_{6}\right]$ que forman cadenas según [100], [010] y [001]. Los iones sodio ocupan además un segundo tipo de posición $\left[\mathrm{Na}^{+}(2)\right]$ de coordinación 8 o 12 con el F, dependiendo de la variedad que se trate. Efectivamente, este compuesto presenta dos variedades polimórficas: puede ser monoclínica " $\mathrm{P} 2 / 1 \mathrm{n}$ " (fase $\alpha$ ), estable en condiciones ambientales $\left(22^{\circ} \mathrm{C}\right)$, o rómbica "Immm" (pseudotetragonal) (fase $\beta$ ) por encima de los $617^{\circ} \mathrm{C}$, presentando entre una y otra toda una serie de fluctuaciones y fases intermedias. El carácter definitivo de esta transición $(\alpha-\beta)$ se estableció recientemente por Yang et al. en 1993 (5). Hasta entonces se habia considerado una transformación monoclínicocúbico que tenía lugar a $550^{\circ} \mathrm{C}$ (6). En las figuras $1 \mathrm{a}$ y $1 \mathrm{~b}$ se muestran las estructuras correspondientes a las dos fases monoclínica y rómbica, respectivamente. Dichas estructuras se han representado con ayuda del programa Cristallographica (7), tomando como base las constantes reticulares determinadas por Yang et al. (5) para diferentes temperaturas. En estas figuras se comprueba como, al disminuir el parámetro c en la variedad de baja temperatura, las cadenas de octaedros $\left[\mathrm{AlF}_{6}\right]$ y $\left[\mathrm{Na}(1) \mathrm{F}_{6}\right]$ que se sitúan en esta dirección, tienen que adoptar una disposición en zig-zag más acorde con las nuevas dimensiones de la celda. Asimismo se observa una importante modificación de los huecos ocupados por el catión $\left[\mathrm{Na}^{+}(2)\right]$ que aparece desplazado con respecto a la fase rómbica y que, además, disminuye su coordinación de 12 a 8 $\left(\left[\mathrm{Na}(2) \mathrm{F}_{12}\right]\right.$ a $\left.\left[\mathrm{Na}(2) \mathrm{F}_{8}\right]\right)$. Finalmente se observa una reducción de la simetría, consistente en la pérdida de dos familias de ejes binarios (coincidentes con [100] y [001]) y sus respectivos planos perpendiculares.

Mediante resonancia magnética nuclear (RMN) se han podido estudiar las diferentes etapas de esta transición en 
función de la variación de temperatura, así como los movimientos asociados a ellas (2). Esta técnica ha permitido comprobar que se trata de una transformación reversible que se produce por la difusión del sodio $\left[\mathrm{Na}^{+}(2)\right]$ a través del armazón de octaedros ([AlF $]$ y $\left.\left[\mathrm{NaF}_{6}\right]\right)$ mediante un mecanismo de movimiento de vacancias. Dicho mecanismo esta facilitado por las oscilaciones que experimentan los octaedros para ajustarse a las distancias interatómicas estables para cada temperatura.

Este tipo de transformación desplazativa, caracterizada por la distorsión espontánea de la estructura y la reducción del grupo puntual de simetría, que se produce al pasar a la forma de baja temperatura, se define como ferroelástica $(8,9)$. Dicha transición origina dominios maclados, cuyos planos límites ocupan la posición de los elementos de simetría perdidos. El objetivo principal de este trabajo es mostrar los dominios maclados que se obtienen experimentalmente en cristales de criolita, crecidos a partir de un fundido.

\section{TRABAJO EXPERIMENTAL}

Se ha utilizado criolita sintética comercial. Su ficha técnica correspondiente revela $\mathrm{Si}$ y Fe como impurezas, en una proporción inferior a 0,5\% y 0,05\%, respectivamente. 30 gr de este producto, finamente pulverizado, se introdujeron en crisoles de Pt-Ir $\left(\mathrm{h}=4 \mathrm{~cm}, \varnothing_{\text {MÁx }}=3,6 \mathrm{~cm}\right.$ y vol. $\left.=30 \mathrm{ml}\right)$ y se cubrieron con una tapa del mismo material. Estos, a su vez, se introdujeron en otros crisoles de porcelana vitrificada, rellenando con alúmina en polvo los espacios vacíos. Finalmente, se cubrió el conjunto con una segunda tapa, de porcelana vitrificada en este caso. La difracción de rayos $\mathrm{X}$ de dicha porcelana nos ha permitido identificar mullita $\left(\mathrm{Al}_{6} \mathrm{Si}_{2} \mathrm{O}_{13}\right)$ como componente mayoritario. El análisis químico realizado mediante energía dispersiva de rayos $\mathrm{X}$ indica además la presencia de $\mathrm{Al}_{2} \mathrm{O}_{3}$. Según la ficha técnica proporcionada por la casa comercial, este material recibe el nombre de Pythagoras, es capaz de resistir temperaturas de hasta $1.200^{\circ} \mathrm{C}$ así como el ataque de todo tipo de ácidos, exceptuando el fluorhídrico.

Los cristalizadores así preparados se sometieron a diferentes condiciones de temperatura-tiempo en el interior de un horno equipado con un sistema de programación y control de rampas. En la figura 2 se representan las dos curvas programadas. Para su confección se consideró el punto de fusión de la criolita a $1.004^{\circ} \mathrm{C}(10)$. En ambas se hizo subir la temperatura hasta $1.100^{\circ} \mathrm{C}$ pero se estableció una gran diferencia en el tiempo de descenso hasta la temperatura ambiente (durante 24 horas en un caso, frente a 160 horas, en el segundo), con el fin de estudiar su incidencia en el proceso y en las características de los cristales formados. La cámara de trabajo en dicho horno estaba protegida con una cabina de seguridad, fabricada con cerámica refractaria, para evitar el deterioro de las resistencias por efecto de posibles vapores corrosivos, procedentes de la descomposición parcial de la criolita que se produce en las proximidades del punto de fusión (10). Estos vapores originan grandes desperfectos en los crisoles de porcelana, asociados siempre a fenómenos de disolución reprecipitación.

Los productos obtenidos fueron separados mecánicamente del crisol y se prepararon láminas delgadas (transparentespulidas) para su estudio. La identificación se realizó mediante difracción de rayos X (DRX), microscopía óptica de transmisión y reflexión, microscopía electrónica de barrido (MEB) y microanálisis por energía dispersiva de rayos $\mathrm{X}$.

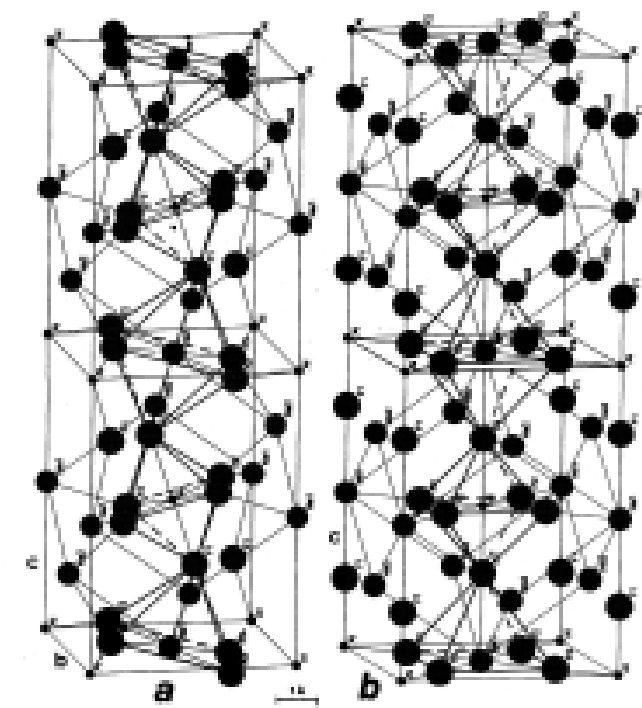

1.- Modelos estructurales de las fases monoclínica " $\mathrm{P} 2_{1} / \mathrm{n}$ " (a) y rómbica "Immm" (b) de la criolita $\left(\mathrm{Na}_{3} \mathrm{AlF}\right)$. En cada una se representa un conjunto de cuatro celdas. [(a) 5.4139:5.6012:7.7769 y $\beta=$ $90.183^{\circ} . \mathrm{T}^{\underline{\mathrm{a}}}=22^{\circ} \mathrm{C}$. (b) $\left.5 \cdot 632: 5 \cdot 627: 7.958 . \mathrm{T}^{\underline{\mathrm{a}}}=617^{\circ} \mathrm{C}\right]$.

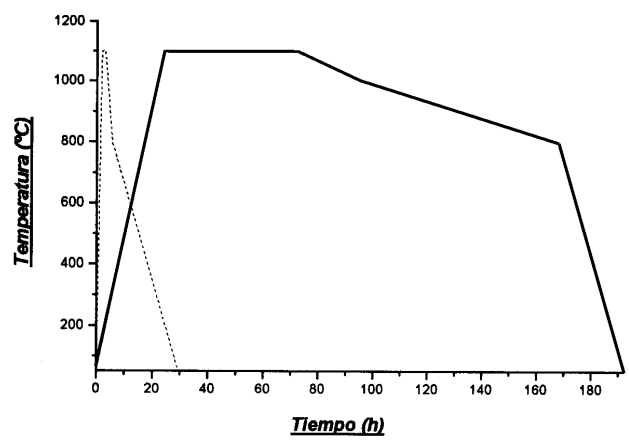

2.- Curvas "temperatura - tiempo" programadas.

\section{RESULTADOS Y DISCUSIÓN}

Mediante la técnica y las condiciones descritas se han obtenido agregados granulares de criolita (identificados mediante DRX, figura 3), con morfologías redondeadas o alargadas, en las que se destacan muy claramente los bordes de grano (figura 4a). Cuando estos agregados se observan con nícoles cruzados (figura 4b), los granos de criolita se comportan como isótropos, debido a su extremadamente débil birrefringencia $(0,0011)$. También destacan especialmente las numerosas maclas polisintéticas que presentan la mayoría de ellos. La figura 5 muestra el aspecto de dichas maclas cuando se observan mediante MEB. El análisis por energía dispersiva de rayos $\mathrm{X}$ en los diferentes dominios maclados confirma que, efectivamente, se trata del mismo material en todos ellos (figura 6). Yang et al (5) establecen que la transición $\alpha$ - $\beta$ de la criolita puede originar dominios maclados, con (100) y (001) como planos límites, y dominios de antifase cuyos límites no están fijados cristalográficamente. Es muy frecuente que los ejemplares naturales presenten el mismo tipo de maclas: "polisintéticas que reflejan el carácter pseudoregular de la celda limitada por $\{001\}$ " (11), que según estas consideraciones, se podrían interpretar como una consecuencia del cambio polimórfico citado y estar determinadas por la historia térmica del mineral (8). 


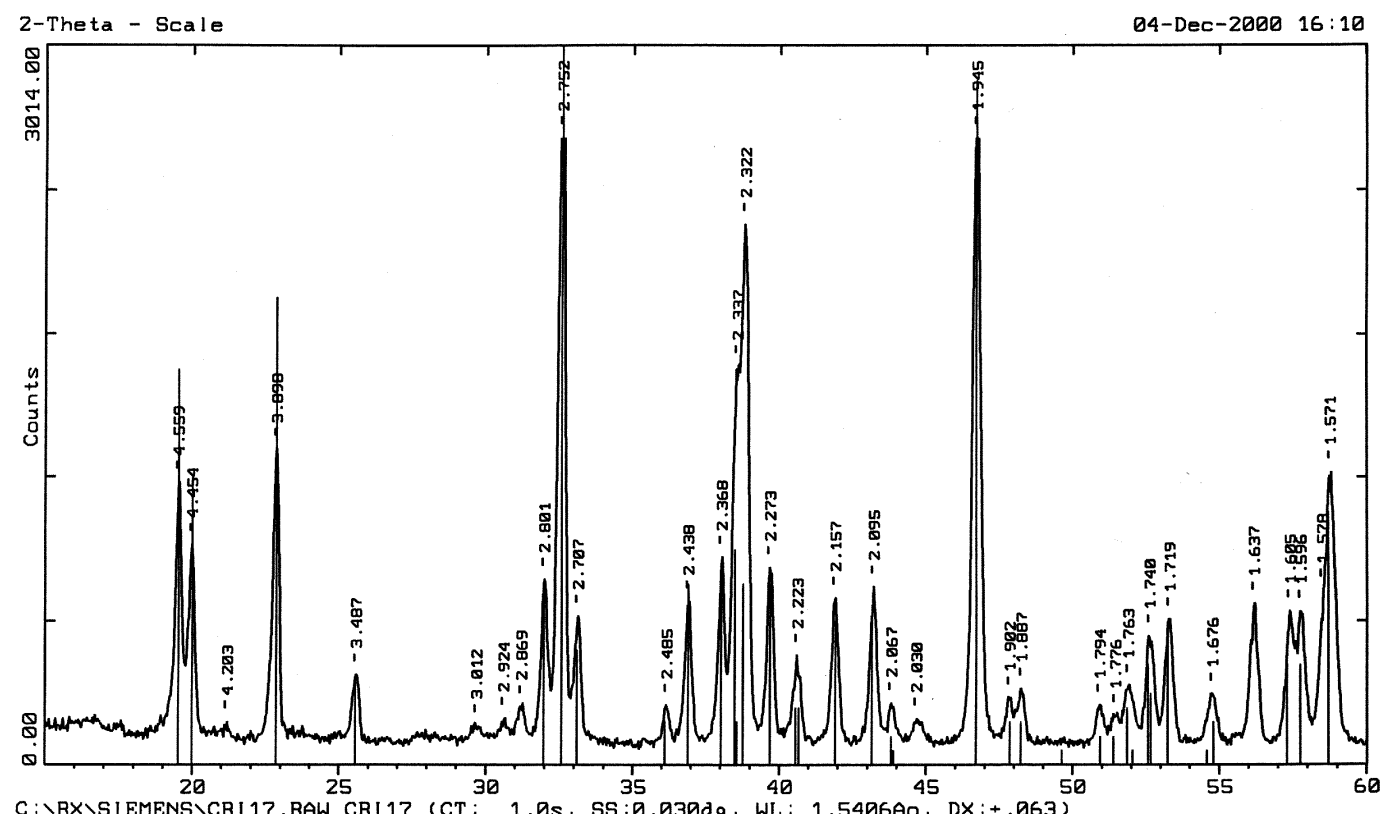

C: \RXISIEMENSICRI 17. RAW CRI17 (CT: $1.0 \mathrm{~S}$, SS:0.030d9, WL: $1.5406 \mathrm{AO}, \mathrm{DX}:+.063$ )

3.- Difractograma de rayos $\mathrm{X}$ correspondiente a uno de los agregados obtenidos. Se identifican criolita “ $\mathrm{Na}_{3} \mathrm{AlF}_{6}$ “ $\mathrm{y}$ villiaumita “ $\mathrm{NaF}^{\prime \prime}(\underline{\underline{2,32}} \underline{\underline{\underline{x}}} \underline{\underline{1,64}} \underline{\underline{6}})$.

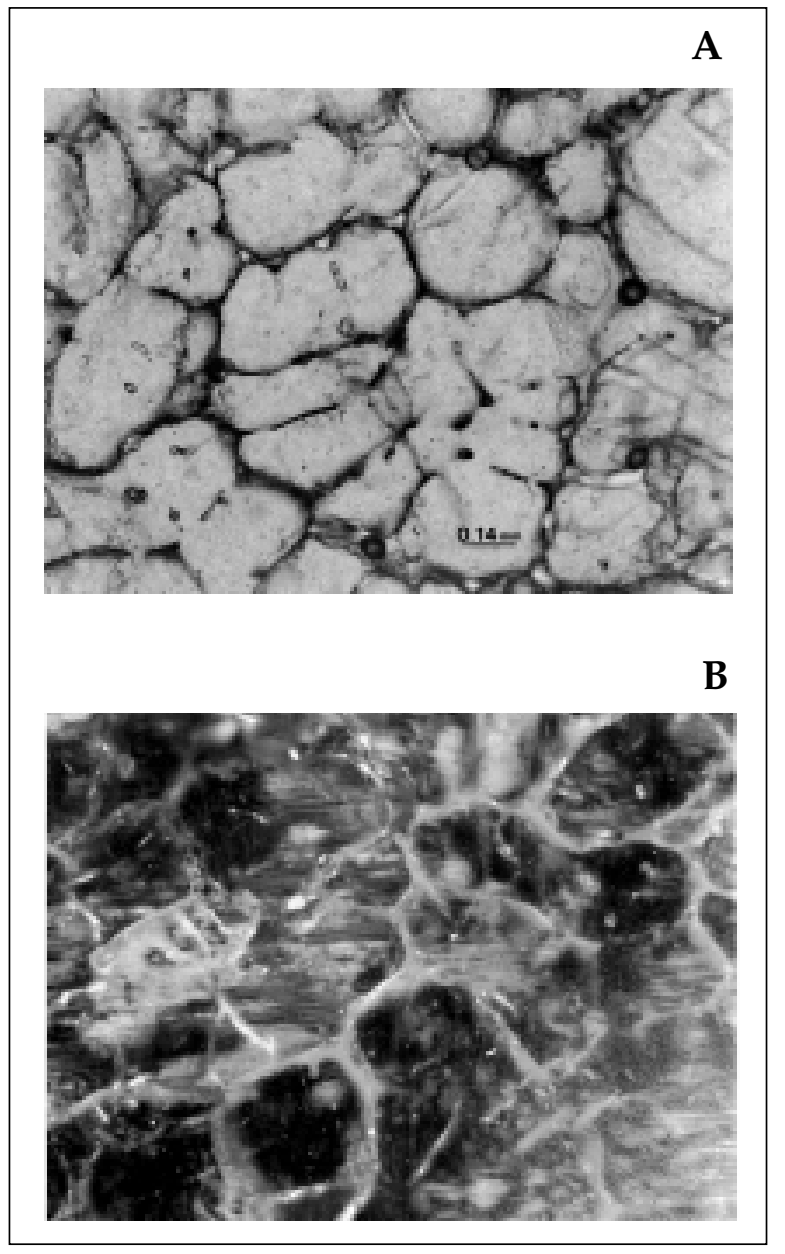

4.- Agregado granular de criolita (microscopía óptica de transmisión X 80). a.- Sin cruzar nícoles. b.- Entre nícoles cruzados. Ver maclas polisintéticas.

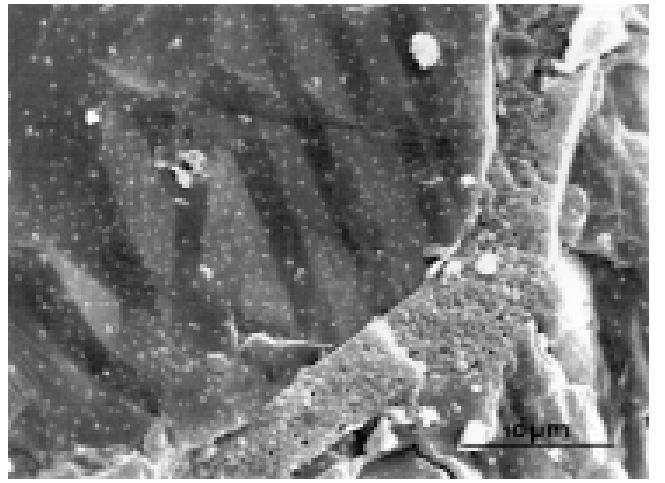

5.- Maclas polisintéticas en un grano de criolita (MEB X 2000).

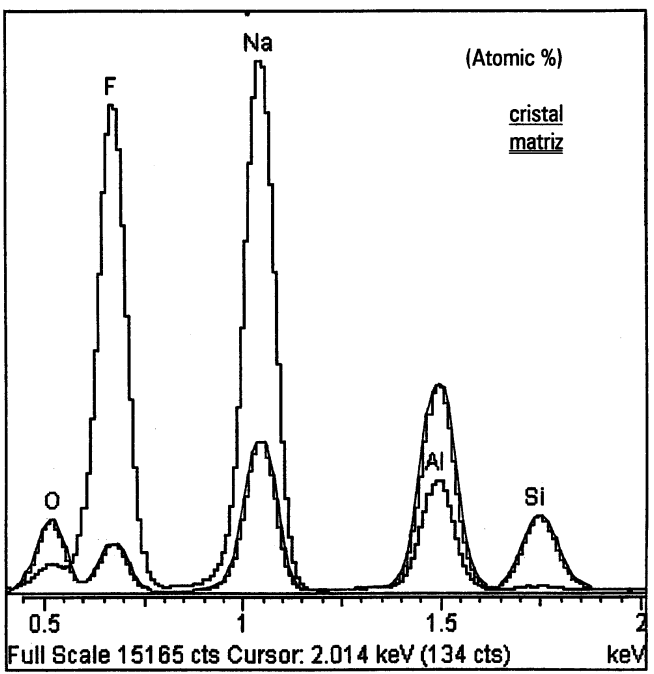

6.- Espectros de energía dispersiva característicos de los granos de criolita (---) y de la matriz que actúa como cemento (=-), respectivamente. 
En el difractograma representado en la figura 3 se identifican, además de la criolita, las dos reflexiones principales de la villiaumita ( $\mathrm{NaF}$ sint. ASTM no 4-793: 2,32 $\underline{x}^{\prime} \underline{1,64}_{6}, 1,34_{2}$ ). Este compuesto ha sido detectado en otros trabajos anteriores (12, 13), donde se utiliza criolita como flujo para la cristalización de corindón. Su presencia se interpreta como el resultado de la descomposición parcial de dicha criolita que tiene lugar en las proximidades de punto de fusión (10), según la expresión: $\mathrm{Na}_{3} \mathrm{AlF}_{6} 2 \mathrm{NaF}+\mathrm{NaAlF}_{4}$.

En relación a los bordes de grano, cuando se observan entre nícoles cruzados, muestran colores amarillos de primer orden (figura 4b). Mediante MEB se ha podido comprobar que dichos bordes consisten realmente en una especie de cáscara que envuelve y separa los diferentes granos (figura 7). El análisis por energía dispersiva de rayos $\mathrm{X}$ indica que dicho envoltorio es un material de naturaleza diferente a la de los granos de criolita que empasta. En él se han identificado Si y O, además de los constituyentes propios de la criolita (figura 6). Esta acumulación de Si en la superficie de los granos de criolita se justifica por la presión de cristalización ejercida por estos durante su desarrollo, que actúa empujando las partículas extrañas que no pueden incorporarse fácilmente a su estructura. Con respecto a la procedencia de éste elemento cabría especular con dos posibilidades, el Si presente como impureza en la criolita, en cantidades tan ínfimas $<0,5 \%$ que no justificaría la elevada proporción detectada en el análisis, o bien, el Si procedente del crisol de cerámica externo, constituido mayoritariamente por mullita $\left(\mathrm{Al}_{6} \mathrm{Si}_{2} \mathrm{O}_{13}\right)$ que, además, muestra signos evidentes de un importante proceso de corrosión.

Finalmente es importante destacar que los agregados descritos presentan las mismas características, independientemente de la rampa aplicada (figura 2), y que su formación se explica, desde el punto de vista del crecimiento cristalino, como un fenómeno de nucleación homogénea primaria, inducido en ambos casos por un sobreenfriamiento excesivamente rápido.

\section{CONCLUSIONES}

A partir de criolita fundida se han obtenido agregados granulares de ésta, como un fenómeno de nucleación homogénea primaria.

Los cristales obtenidos muestran maclas polisintéticas que resultan de la transformación ferroelástica que se produce al descender la temperatura del fundido hasta la temperatura ambiente.

El tiempo aplicado no influye apreciablemente en las características de los agregados ni de las maclas presentes en los cristales.

Durante el proceso tiene lugar la descomposición parcial de la criolita. Esta puede originar:

a.- Fases sólidas como la villiaumita detectada en el residuo sólido

b.- Vapores corrosivos que se desprenden y actúan sobre la porcelana que envuelve externamente a los cristalizadores y que se revela como el principal agente contaminante del sistema.

El Si contaminante se concentra en la superficie de los granos de criolita merced a la presión de cristalización ejercida por estos durante su desarrollo.

\section{AGRADECIMIENTOS}

Este trabajo ha sido financiado por la DGYCIT, proyecto $\mathrm{n}^{\mathrm{o}}$ PB96-0619. El Dr. D. Martín Martínez-Ripoll nos ha facilitado

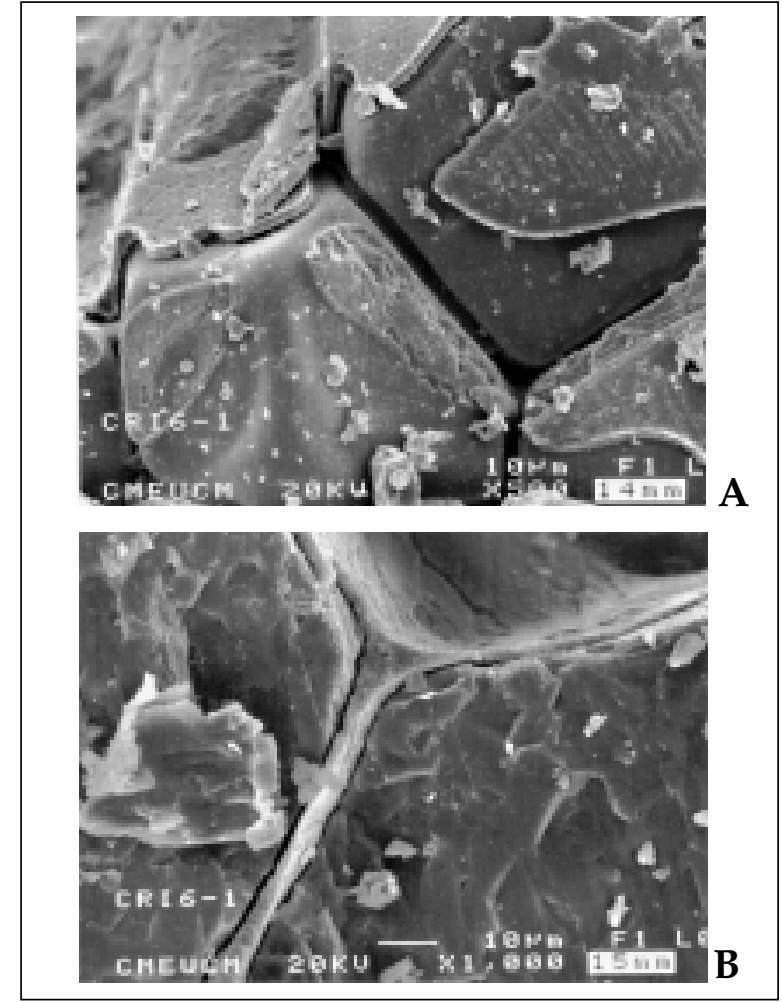

7.- Granos de criolita envueltos por una matriz de diferente naturaleza (MEB). a.- La matriz no aparece entre los dos granos centrales (X 500). b.- Detalle de la matriz (X 1000).

numerosos datos para la representación de estructuras. La preparación de láminas delgadas ha sido realizada por D. Manuel Mora. La microscopía electrónica de barrido se ha realizado con la colaboración de D. Alfonso Rodriguez Muñoz. Las observaciones y sugerencias del Dr. D. Roberto Oyarzun han contribuido a mejorar este trabajo.

\section{BIBLIOGRAFIA}

1. J. A. Babor y J. Ibarz; Química General Moderna, pp 754. Ed. Marín, Barcelona (España) 1958

2. D. R.Spearing, J. F. Stebbins \& I. Farnan; Diffusion and the dynamics of displacive phase transitions in cryolite $\left(\mathrm{Na}_{3} \mathrm{AlF}_{6}\right)$ and chiolite $\left(\mathrm{Na}_{5} \mathrm{Al}_{3} \mathrm{~F}_{14}\right)$ : multi-nuclear NMR studies; Phys. Chem. Minerals, 21, 373-386 (1994)

3. H. Pauly; Mechanical properties of cryolite from Ivigtut, South Greenland; Bull. Geol. Soc. Den. 33, 401-413 (1985)

4. E. W. Dewing; Solid solubility of $\mathrm{CaF}_{2}$ in cryolite; Trans. TMS-AIME 245, 1829-1830 (1969)

5. H. Yang, S. Ghose, D. M. Hatch; Ferroelastic phase transition in cryolite, $\mathrm{Na}_{3} \mathrm{AlF}_{6^{\prime}}$ a mixed fuoride perovsite: high temperature simple crystal X-ray diffraction study and symmetry analysis of the transition mechanism; Phys. Chem. Minerals, 19, 528544 (1993)

6. E. G. Steward, H. P. Rooksby; Transitions in crystals structure of cryolite and related fluorides; Acta. Cryst. 6, 49 (1953)

7. Crystallographica V.1.51. Oxford Cryosystems. U.K.

8. A. Putnis; Introduction to Mineral Science, pp 223-225. Cambridge University Press, New York (EEUU) 1992

9. E. K. H. Salje; Mesoscopic Twin Patterns in Ferroelastic and Co-elastic Minerals, pp 65-84. Reviews in Mineralogy \& Geochemistry. Vol 39 Washington (U.S.A.) 2000

10. A. P. Foster; Cryolite-Alumina phase diagram by quenching methods. J. Am. Ceram. Soc. 43 (2) 66-67 (1960)

11. C. Klein, S. C..Hurlbut JR; Manual de Mineralogía, $4^{a}$ ed. pp. 444. Editorial Reverte, Barcelona (España) 1997

12. C. Prieto Colorado, E. Fernández Sánchez, V. López-Acevedo y S. López Andrés, Microraman analysis of corundum crystallized by criolite flux, pp. 83-84. 4 Th International Conference on Raman Spectroscopy Applied to the Earth Sciences. Serie: Ciencias no 19 . Ed.: Universidad de Valladolid (España) 1999.

13. V. López-Acevedo; Cristalización de gemas en solución de alta temperatura: técnica del flujo. Boletín de la Real Sociedad Española de Historia Natural. Sección Geológica. Vol. 96 (En prensa). 